\title{
Antibiotic Susceptibility Pattern and Molecular Typing By PCR-RAPD Analysis of Clinical and Environmental Isolates of Pseudomonas aeruginosa
}

\author{
O. B. Oluborode' ${ }^{1}$, S.I. Smith ${ }^{2 *}$, T.A. Seriki ${ }^{3}$, M. Fowora ${ }^{2}$, A. Ajayi ${ }^{3}$, and A.O. Coker ${ }^{4}$ \\ ${ }^{1}$ Department of Medical Microbiology and Parasitology, University of Lagos, Idi-Araba, Nigeria \\ ${ }^{2}$ Molecular Biology \& Biotechnology Department, Nigerian Institute of Medical Research (NIMR), Yaba, Lagos, Nigeria \\ ${ }^{3}$ Department of Microbiology, University of Lagos Akoka, Nigeria \\ ${ }^{4}$ Babcock University Teaching Hospital, Ogun State, Nigeria
}

Received: May 22, 2018 / Revised: September 10, 2018 / Accepted: September 11, 2018

Pseudomonas aeruginosa accounts for a significant proportion of nosocomial infections. This study examined the antimicrobial susceptibility pattern and clonal relatedness of $P$. aeruginosa isolates of clinical and environmental origin. These isolates displayed susceptibility to levofloxacin, ciprofloxacin, gentamicin, imipenem, and ceftazidime of $65.0 \%, 62.5 \%, 90.0 \%, 100 \%$, and $85 \%$, respectively. PCR-RAPD analysis of the $P$. aeruginosa isolates revealed marked variation. No correlation was observed between the antibiotic resistance profiles and the DNA typing patterns.

Keywords: Resistance, PCR-RAPD, Pseudomonas aeruginosa, clonal relatedness

Referred to as a panacea to cure infections, antibiotics have been greatly relied upon by humans in combating infections for over 60 years. However the emergence of resistance by pathogenic bacteria most especially multidrug resistance (MDR) has remained a great threat to the use of these miracle molecules in medical practice [1]. MDR bacteria abound in the environment and have been implicated in human infections. One of such bacterium is Pseudomonas aeruginosa an opportunistic pathogen associated with nosocomial infections mostly in patients with impaired immunity [2, 3]. P. aeruginosa exhibits a remarkable antibiotic resistance profile as it has an extraordinary ability of acquiring further mechanisms of resistance to multiple groups of antibiotics outside its natural resistance [4]. In Nigeria, several studies have shown high resistance of $P$. aeruginosa obtained

\section{*Corresponding author}

Tel: +234 8037058989

E-mail: stellaismith@yahoo.com

○ 2018, The Korean Society for Microbiology and Biotechnology from both clinical and environmental sources to various classes of antibiotics most especially those isolated from hospital settings [5-9]. It is therefore imperative to determine the genetic relatedness of $P$. aeruginosa isolated from clinical samples and those isolated from the environment by genotyping, since typing of strains is necessary for determining the epidemiology of nosocomial infections and in the design of pathogen control strategies [10]. This study investigates the antimicrobial susceptibility pattern of clinical and environmental isolates of Pseudomonas aeruginosa and the clonal relatedness of these species.

Forty eight isolates of clinical (30) and environmental (18) species of Pseudomonas obtained from the Molecular Biology and Biotechnology Laboratory of the Nigerian Institute of Medical Research (NIMR) were subjected to antibiotic susceptibility testing using the disc diffusion method according to the clinical laboratory standards Institutes (CLSI) guidelines [11]. Identification and clonal relatedness of isolates was determined by poly- 
Table 1. Genus and species specific Pseudomonas PCR primers and RAPD primers.

\begin{tabular}{ll}
\hline \multicolumn{1}{c}{ Primers } & \\
\hline $\begin{array}{ll}\text { Pseudomonas } \\
\text { specific }\end{array}$ & (PA-GS)F 5'-CACTGGTGTTCCTTCCTATA-3' [12] \\
& (PA-GS)R 5'-GACGGGTGAGTAATGCCTA-3' \\
& (PA-SS)R 5'-GCCGGATCTTCGGACCTCA-3' \\
RAPD & (10514) 5' TGGTGGCCTCGAGCAAGAGAACGG-3' [13] \\
& (14306) 5'-GGTTGGGTGAGAATTGC-3' \\
\hline
\end{tabular}

merase chain reaction (PCR) and random amplified polymorphic DNA analysis (RAPD) using pairs of primers shown in Table 1. Using Pseudomonas genus-specific primers PA GS-F and PA GS-R 30 clinical (62.5\%) and 18 environmental (37.5\%) isolates were identified to be Pseudomonas species. Of these 48 Pseudomonas species, P. aeruginosa-specific primers PA SS-F and PA SS-R confirmed 40 to be Pseudomonas aeruginosa comprising 26 clinical (54.17\%) and 14 environmental (29.17\%) isolates. RAPD analysis of the 40 Pseudomonas aeruginosa isolates produced 8 clusters consisting of 37 different PCR-RAPD profile (Fig. 1). PCR based molecular typing methods have been suggested for epidemiological typing of $P$. aeruginosa strains, among which RAPD typing has been recommended as an efficient tool in clonal discrimination of this pathogen [14]. Molecular typing by PCRRAPD analysis of $P$. aeruginosa isolates from clinical and environmental sources resulted in thirty-seven different PCR-RAPD profiles. This showed high level of variations based on the banding patterns among the isolates and agrees with the expectation from an organism with a much larger genome [15]. Of the eight clusters generated, cluster 1 comprised sixteen isolates, four of which were clonally related. Other clusters comprised of two to four isolates which were probably not clonally related. This shows that there is a high rate of polymorphism and hyper-mutation in $P$. aeruginosa genome. High level of genetic diversity in $P$ aeruginosa isolates has also been reported as the basis of $P$. aeruginosa multidrug resistance and high tenacity in environmental adaptability [14]. However, there seems to be no relationship between the genetic profiles and antibiotic susceptibility patterns of the isolates since no correlation was observed between the antibiotic resistance profiles and the DNA subtyping patterns. Similar results have been reported in studies by Smith et al. [6], and Sinem and Nilufer [16], who observed no relationship between the antibiotic susceptibility profiles and molecular typing patterns of $P$. aeruginosa.

Antibiotic susceptibility pattern and profile of Pseudomonas aeruginosa against test antibiotics are indicated in Table 2. Twenty six (65\%), were susceptible to levofloxacin, 27 (62.5\%) were susceptible to ciprofloxacin, 36 (90\%) were sensitive to gentamicin, 40 (100\%) were sensitive to imipenem, while 34 (85\%) were sensitive to ceftazidime. Comparison of the sensitivity of environmental and clinical isolates to test antibiotics showed that clinical isolates were more sensitive to levofloxacin and ciprofloxacin, while both environmental and clinical isolates were all sensitive to imipenem (Table 2). In the present study $P$. aeruginosa isolates from environments were less sensitive to ceftazidime, levofloxacin and ciprofloxacin than the clinical isolates, while sensitivity to gentamicin was higher in environmental isolates than clinical. However, the observed differences was not statistically significant $(p>0.05)$ : ciprofloxacin $(p=0.750)$, gentamicin $(p=0.658)$ and ceftazidime $(p=0.403)$ except levo-

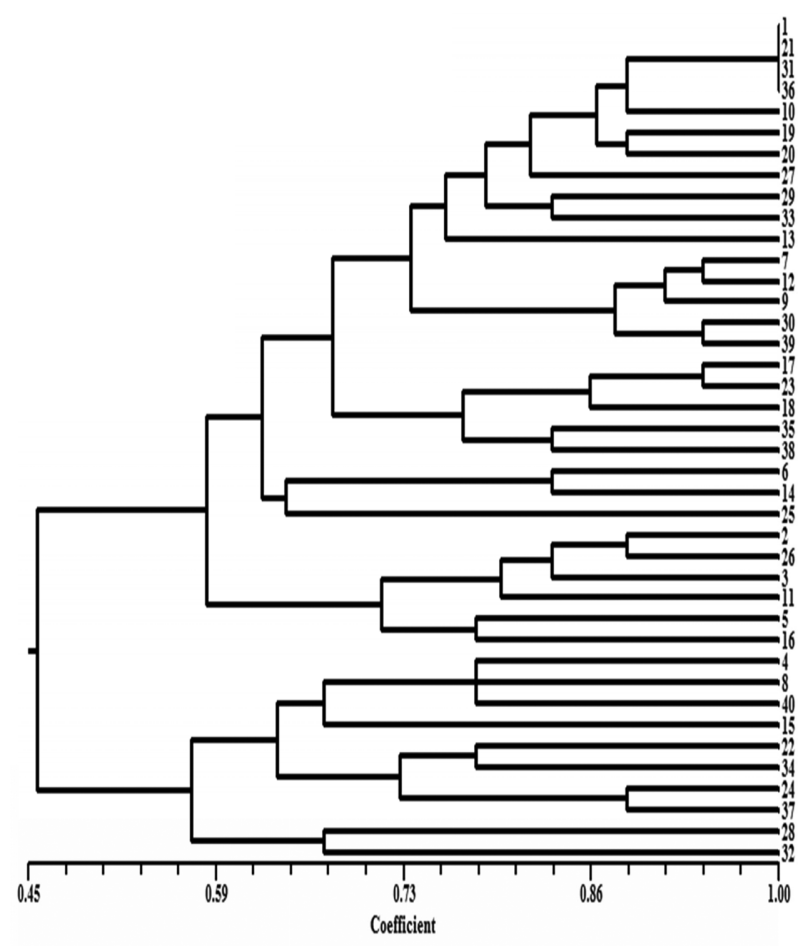

Fig. 1. Dendrogram constructed by UPGMA showing clustering of Pseudomonas aeruginosa based on RAPD. I to VIII stand for the eight clusters that were generated from the dendrogram at the co-efficient of similarity. 
Table 2. Antimicrobial susceptibility pattern of the Pseudomonas aeruginosa isolates.

\begin{tabular}{lcccc}
\hline Antibiotics & $\begin{array}{c}\text { Clinical sensitive isolates. } \\
\mathrm{N}(\%) n=26\end{array}$ & $\begin{array}{c}\text { Environmental sensitive } \\
\text { isolates. N (\%) } n=14\end{array}$ & $\begin{array}{c}\text { Total number of sensitive } \\
\text { isolates. N (\%) } n=40\end{array}$ & $p$-values \\
\hline Levofloxacin & $20(76.92)$ & $6(42.85)$ & $26(65.0)$ & 0.031 \\
Ciprofloxacin & $18(69.23)$ & $9(64.28)$ & $27(62.5)$ & 0.750 \\
Gentamicin & $23(88.46)$ & $13(92.85)$ & $36(90.0)$ & 0.195 \\
Imipenem & $26(100)$ & $14(100)$ & $40(100)$ & - \\
Ceftazidime & $23(88.46)$ & $11(78.57)$ & $34(85)$ & 0.403 \\
\hline
\end{tabular}

floxacin, which had a $p$-value of 0.031 . Imipenem was found to be highly sensitive (100\%) against both clinical and environmental isolates. This corroborates the finding of Chika et al. [17], and Odumosu et al. [18], who reported $P$. aeruginosa isolates from environmental, clinical, human, animals and plants not resistant to imipenem. The potency of this antibiotic could be as a result of its conserved usage for only life threating cases. However there is need for caution in the use of this molecule since resistance in $P$. aeruginosa has been reported in Brazil [19]. Susceptibility (90\%) of $P$. aeruginosa to gentamicin was next to imipenem. This is in line with reports from Lagos [20] and Ethiopia [21] of 91.2\% and 91.7\% susceptibility of $P$. aeruginosa to gentamicin respectively. Hence gentamicin is one aminoglycoside which could still be considered a potent antibiotic in the treatment of infections caused by $P$. aeruginosa [22]. There was $85 \%$ susceptibility of $P$. aeruginosa to ceftazidime, a third generation cephalosporin. This concurs with reports of $85.25 \%$ and $88.3 \%$ susceptibility by Odumosun et al. [23] and Olayinka et al. [24]. However there was reduced susceptibility of $P$. aeruginosa to fluoroquinoles compared to earlier studies in Nigeria (Odumosun et al. [23]; Mohammed et al. [25] and Osundiya et $a l$. [20]). Findings from this study show that imipenem, gentamicin and ceftazidime are still effective antimicrobials against $P$. aeruginosa therefore prudent use of these molecules is advocated to forestall development of resistance.

\section{Conflict of Interest}

The authors have no financial conflicts of interest to declare.

\section{References}

1. World Health Organization. Antimicrobial resistance 2014. Global Report on Surveillance. WHO press, World Health Organi- zation, 20 Avenue Appia 1211 Geneva 27, Switzerland. www.who. int. Accessed Feb. 20, 2018.

2. Olga P, Apostolos V, Alexis G, George V, Athena M. 2016. Antibiotic resistance profiles of Pseudomonas aeruginosa isolated from various Greek aquatic environments. FEMS Microbiol. Ecol. 92: 1-7.

3. Dantas RCC, Silva RT, Ferreira ML, Goncalves IR, Araujo BF, de Campos PA, et al. 2017. Molecular epidemiology survey of bacteremia by multidrug resistant Pseudomonas aeruginosa: The relevance of intrinsic resistance mechanisms. PLoS One 12: e0176774.

4. Strateva T, Yordanov D. 2009. Pseudomonas aeruginosa a phenomenon of bacterial resistance. J. Med. Microbiol. 58: 11331148.

5. Chikwendu Cl, Amadi ES, Obi RK. 2010. Prevalence and antimicrobial resistance in Pseudomonas aeruginosa and Klebsiella pneumoniae isolate from non-clinical urine samples. NY. Sci. J. 3: 194-200.

6. Smith S, Ganiyu O, John R, Fowora M, Akinsinde K, Odeigah P. 2012. Antimicrobial resistance and molecular typing of Pseudomonas aeruginosa isolated from surgical wounds in Lagos Nigeria. Acta. Medica. Iranica. 50: 433-438.

7. Eyo A-AO, Ibeneme EO, Thumamo BDP, Asuuo AE. 2015. Antibiotic resistance profile of clinical and environmental isolates of Pseudomonas aeruginosa in Calabar, Nigeria. IOSR J. Pharm. Biol. Sci. 10: 9-15.

8. Adesoji AT, Ogunjobi AA, Olatoye IO. 2015. Molecular characterization of selected multidrug resistant Pseudomonas from water distribution systems in South-Western Nigeria. Ann. Clin. Microbiol. Antimicrob. 14: 39.

9. Igbalajobi OA, Oluyege AO, Oladeji AC, Babalola JA. 2016. Antibiotic resistance pattern of Pseudomonas aeruginosa isolated from clinical samples in Ekiti State University Teaching Hospital AdoEkiti, Ekiti state of Nigeria. Br. Med. Res. J. 12: 1-6.

10. Al-Haik, WM, Al-Mahbash, AA, Al-Mahdi, AY, Mohamed, MME, AlHaddad, AM. 2016. Genotypic characteristics of clinical and nonclinical isolates of Pseudomonas aeruginosa: Distribution of different antibiogram profiles and molecular typing. Jordan J. Biol. Sci. 9: 185-194.

11. Clinical and Laboratory Standards Institute. Performance Standards for Antimicrobial Susceptibility Testing; Twenty-Third Informational Supplement. 2013. 33: M100-S23.

12. Spilker T, Coenye T, Vandamme P, Lipuma JJ. 2004. PCR-based assay for differentiation of Pseudomonas aeruginosa from other 
Pseudomonas species recovered from cystic fibrosis patients. J. Clin. Microbiol. 42: 2074-2079.

13. Kersulyte D, Struelens MJ, Deplano A, Berg DE. 1995. Comparison of arbitrarily primed PCR and microrestriction (Pulsed Field Gel Electrophoresis) typing of Pseudomonas aeruginosa strains from cystic fibrosis patients. J. Clin. Microbiol. 33: 2216- 2219.

14. Nazik H, Ongen B, Erturan Z, Salcioglu M. 2007. Genotype and antibiotic susceptibility patterns of $P$. aeruginosa and Stenotrophomonas maltophila isolated from cystic fibrosis patients. Japan J. Infect. Dis. 60: 82-86.

15. Akanji BO, Ajele JO, Onasanya A. 2011. Genetic fingerprinting of Pseudomonas aeruginosa involved in nosocomial infection as revealed by RAPD-PCR markers. Biotechol. 10: 70-77.

16. Sinem DG, Nilufer A. 2016. Molecular typing of clinical Pseudomonas aeruginosa strains by using RAPD-PCR. Minerva Biotecnologica 28: 104-13.

17. Chika EO, Nnek AR, Dorothy ON, Chika E. 2017. Multidrug resistant Pseudomonas aeruginosa isolated from hospitals in Onitsha South-Eastern Nigeria. Int. Archives Biomed. Clin. Res. 3: 22-26.

18. Odumosu BT, Ajetunmobi O, Dada-Adegbola H, Odutayo I. 2016. Antibiotic susceptibility pattern and analysis of plasmid profiles of Pseudomonas aeruginosa from human, animal and plant sources. Springerplus 5: 1381. DOI 10.1186/s40064-016-3073-9.

19. Lucia A, de Freitas P, Barth AL. 2002. Antibiotic resistance and molecular typing of Pseudomonas: Focus on imipenem. Braz. J.
Infect. Dis. 6: 1-7.

20. Osundiya OO, Oladele RO, Oduyebo OO. 2013. Multiple Antibiotic Resistance (MAR) indices of Pseudomonas and Klebsiella species isolates in Lagos University Teaching Hospital. Afr. J. Clin. Exp. Microbiol. 14: 164-168.

21. Gedamu S, Tilahun G, Bitew M, Terefe G. 2014. Drug sensitivity of Pseudomonas aeruginosa from wound infections in Jimma University Specialized Hospital, Ethiopia. Online J. Med. Med. Sci. Res. 3: 13-18.

22. Okon K, Agukwe P, Oladosu W, Balogun S, Uba A. 2009. Antibiotic resistance pattern of Pseudomonas aeruginosa isolated from clinical specimens in a tertiary hospital in Northeastern Nigeria. Int. J. Microbiol. 8: 1-6.

23. Odumosu BT, Adeniyi BA, Soge OO, Dada-Adegbola H. 2012. Multidrug resistant Pseudomonas aeruginosa from South-West Nigeria hospitals. Int. J. Pharm. Sci. Rev. Res. 15: 11-15.

24. Olayinka AT, Olayinka BO, Onile BA. 2009. Antibiotic susceptibility and plasmid pattern of Pseudomonas aeruginosa from the surgical unit of a university teaching hospital in North-Central Nigeria. Int. J. Med. Med. Sci. 1: 79-83.

25. Mohammed A, Adeshina GO, Ibrahim YK. 2013. Incidence and antibiotic susceptibility pattern of bacterial isolates from wound infections in a Tertiary Hospital in Nigeria. Trop. J. Pharm. Res. 12: 617-621. 